Bodenbelägen statt mit Teppichen ausgelegt. Schließlich gaben mehr Mütter als in der Vergleichsgruppe an, mit dem Zufüttern von Obst und Gemüse bis zu einem Alter des Kindes von 26 Wochen zu warten. Nur die Forderung, bereits während der Schwangerschaft auf das Rauchen zu verzichten und die Kinder nach Möglichkeit länger als 6 Monate zu stillen, wurde von allergischen Müttern nicht häufiger beherzigt.

Insgesamt waren die Autoren der Studie jedoch überrascht, wie stark die allergischen Eltern bemüht sind, eine allergenarme Umgebung für sich und ihr Kind zu schaffen. Ob dies tatsächlich gelingt, dürften die Ergebnisse der Allergenmessungen zeigen, die im Alter von drei Monaten durchgeführt wurden, deren Ergebnisse jedoch noch nicht vorliegen.

\section{Fazit}

Wegen der großen Teilnehmerzahl von 3.14 Kindern verspricht PIAMA wertvolle Erkenntnisse zum Einfluss der Allergenvermeidung auf das Allergierisiko zu liefern. Der Aussagewert zum genetischen Risiko wird aufgrund der - aus präventiver Sicht sicher gewünschten Lebensstilanpassung von Allergikern wohl begrenzt bleiben.

rme

Wijga A et al. Are children at familial risk of developing allergy born into a low risk environment? The PIAMA birth cohort study. Clin Exp Allergy 2001; 31: 576-81

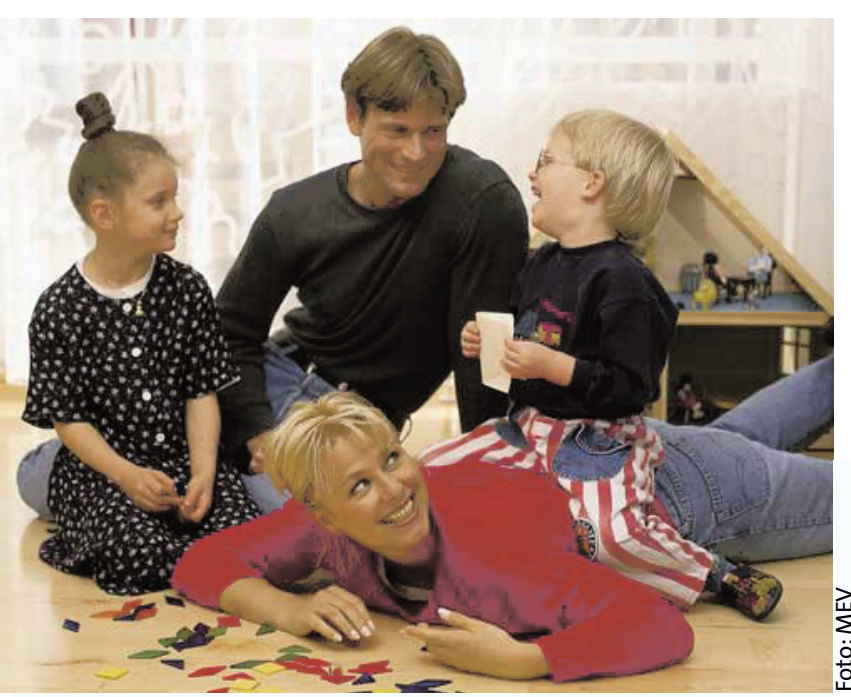

Keine Allergien in der Familie - einfach nur Glück gehabt oder liegt's an der konsequenten Allergenreduktion?

\title{
Allergenreduktion: viel Aufwand, wenig Wirkung?
}

\section{Eine Expositionsprophylaxe gegenüber Umweltallergenen scheint einen realistischen Ansatz zur primären Allergieprävention dar- zustellen. In einer neuen prospektiven Studie sollte nun untersucht werden, ob eine allergenarme Umwelt bei Kindern zu einem ver- minderten Auftreten von Asthma oder atopischem Ekzem führt.}

n der Studie nahmen 291 Paare teil, die als Hochrisikogruppe eingestuft wurden, da beide Elternteile atopisch waren; sie durften kein Haustier halten. Vergleichsgruppen waren 161 Kinder mit zwei atopischen Eltern mit Haustieren sowie 168 Kinder, bei deren Eltern keine atopische Diathese bestand und kein Tier im Haushalt vorhanden war. Von 145 Paaren der Studiengruppe wurden Maßnahmen zur Reduktion des Allergengehalts der Wohnräume durchgeführt, 146 Paare dienten als Kontrollgruppe.

Als Maßnahmen zur Allergenreduktion erhielten die Teilnehmer der aktiv präventiv behandelten Gruppe in der 16. Schwangerschaftswoche Milbenallergenschutzbezüge für das Bett der Mutter sowie Staubsauger mit besonderen Filtern. In der 36. Schwangerschaftswoche wurden Teppiche aus dem Kinderzimmer entfernt und ein VinylBelag verlegt. Die Paare erhielten milbenallergendichte Matratzen für das Kinderbett und waschbare Stofftiere. Sie wurden gebeten, die Bettwäsche des Kindes einmal wöchentlich heiß $\mathrm{zu}$ waschen. Im übrigen Haushalt kam bei Teppichen und Polstermöbeln zur Milbensanierung Benzylbenzoat zum Einsatz.

Die Autoren der Studie halten als Ergebnis fest, dass bei Kindern der Interventionsgruppe im Vergleich zu den Kindern der Studiengruppe ohne aktive Präventionsmaßnahmen im ersten Lebensjahr $\sum_{i}$ das relative Risiko für Gieơ men oder Atemnot auf 0,44 (95\%-Konfidenzintervall 0,20-1,00), für den
Gebrauch von verschreibungspflichtigen Medikamenten zur Behandlung der Atemnot auf 0,58 (95\%-Konfidenzintervall 0,36-0,95) sowie für das Auftreten von Anstrengungsasthma auf 0,18 (95\%-Konfidenzintervall 0,04-0,79) reduziert war. Die Wahrscheinlichkeit für das Auftreten von Atemwegssymptomen war in der Kontrollgruppe und in der Gruppe von Hochrisikopatienten mit Haustieren vergleichbar.

Unterschiede in der Häufigkeit eines atopischen Ekzems konnten zwischen der Gruppe mit umfangreichen Allergenschutzmaßnahmen, der Kontrollgruppe und der Hochrisikogruppe mit Haustieren nicht festgestellt werden. Einzig bei den Kindern mit niedrigem Risiko für eine atopische Erkrankung war das Auftreten von Ekzemen deutlich seltener. Bei Kindern nicht atopischer Eltern ging der Besitz einer Hauskatze signifikant mit einer Sensibilisierung gegenüber Katzenallergenen einher.

\section{Fazit}

Die Autoren gelangen zu dem Schluss, dass intensive Präventionsmaßnahmen in gewissem Umfang respiratorische Symptome bei Kindern mit hohem Risiko für atopische Erkrankungen mindern. Weitere Untersuchungen sind nötig, um zu belegen, daß eine allergenarme Umwelt tatsächlich auch hier definitiv protektiv ist. Alles in allem sind die Ergebnisse dieser präventiven Therapie eher als marginal zu werten, insbesondere unter Berücksichtigung des betriebenen Aufwandes! A. Niedermeier, München

Custovic A. et al. Effect of environmental manipulation in pregancy and early life on respiratory symptoms and atopy during first year of life: a randomised trial. Lancet 2001; 358: 188-93 